Department of

Neurology, Atkinson

Morley's Hospital, St

George's Hospital NHS

Trust, Copse Hill,

Wimbledon, London

SW20 ONE, UK

$\mathrm{H}$ Modarres

M Samuel

F Schon

Correspondence to:

Dr H Modarres

Received 15 April 1999 and in revised form

1 November 1999

Accepted 14 December 1999

\section{Isolated finger flexion: a novel form of focal neuromyotonia}

\author{
H Modarres, M Samuel, F Schon
}

\begin{abstract}
Two almost identical elderly women are described who presented with gradually progressive painless involuntary flexion of the ring and middle fingers over 12 months, leading eventually to contractures. The flexion deformity persisted
\end{abstract}
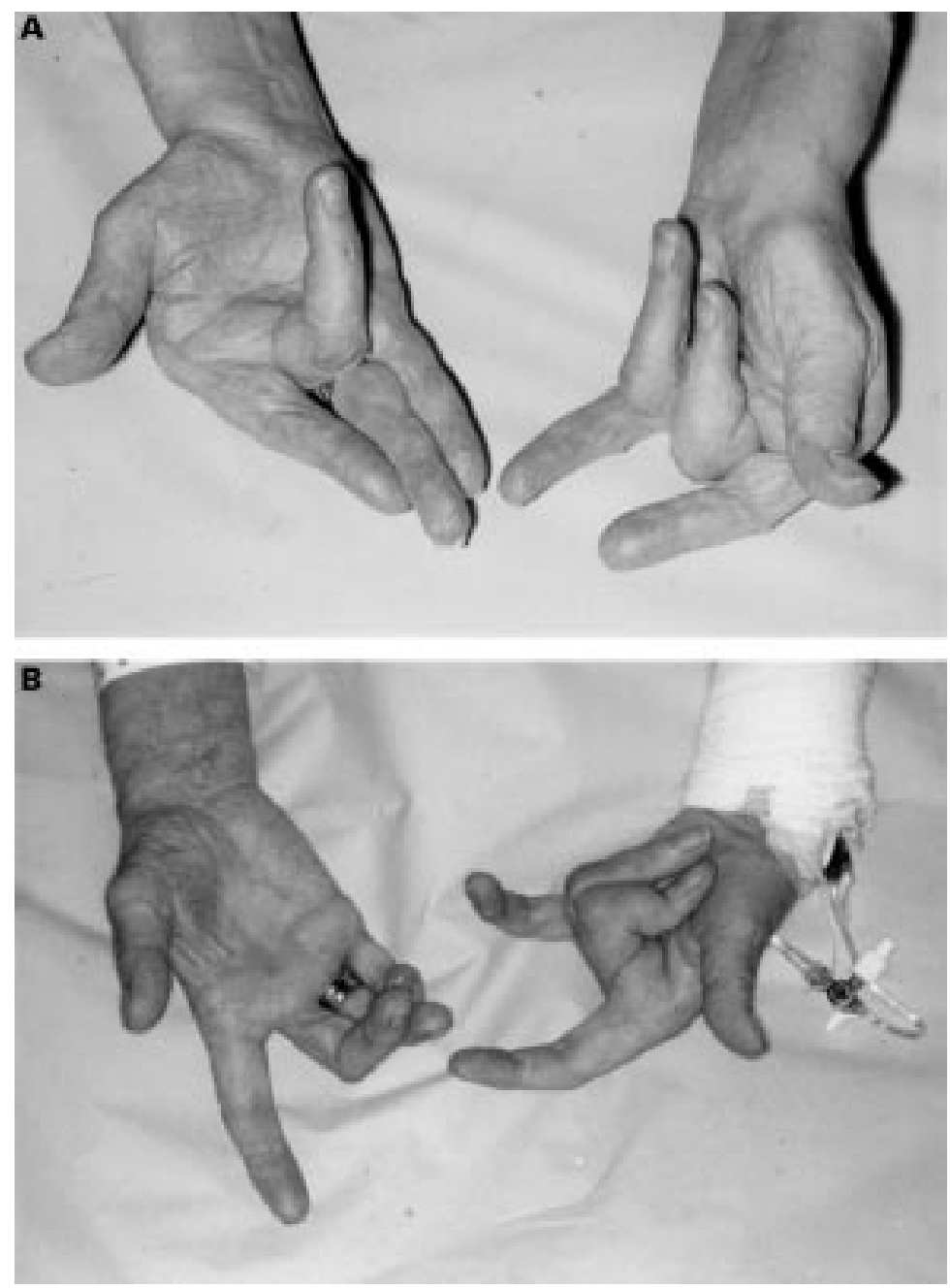

Figure 1 Resting position of hands. (A) Patient 1. There is pronounced flexion of the metacarpophalangeal and proximal interphalangeal joints of the left middle and ring fingers and right middle finger. The left middle finger could only be extended passively at the proximal interphalangeal and metacarpophalangeal joints up to $30^{\circ}$ due to contracture, other fingers could be fully extended passively. (B) Patient 2. There is maximum flexion of the metacarpophalangeal and proximal interphalangeal joints of the left middle and ring fingers, which were passively extendable at the proximal interphalangeal joints and only up to $20^{\circ}$ at the metacarpophalangeal joints due to contracture. The right middle finger shows $90^{\circ}$ flexion at the proximal interphalangeal joint, which could be fully extended passively. during sleep and was the sole neurological abnormality. Both patients had advanced chronic obstructive pulmonary disease and were on long term salbutamol and oxygen. Neurophysiological studies indicated that this was due to neuromyotonia mainly involving flexor digitorum superficialis muscles without evidence of underlying peripheral neuropathy, proximal conduction block, or generalised neuromyotonia. Voltage gated potassium channel antibodies were negative. The clinical and neurophysiological picture remained static over a 2 year follow up period. It is suggested that this is a novel form of acquired focal neuromyotonia and speculate both on its cause and distribution. (F Neurol Neurosurg Psychiatry 2000;69:110-113)

Keywords: focal neuromyotonia; involuntary finger flexion; finger contracture

Persistent abnormal finger posture may be due to Dupuytren's contracture and rheumatological diseases. Neurological causes include extrapyramidal disorders, peripheral nerve trauma, and generalised neuromyotonia. ${ }^{12}$

We describe an apparently novel clinical phenomenon characterised by flexion deformity of the middle and ring fingers due to isolated focal neuromyotonia in two elderly patients.

\section{Case 1}

A 78 year old woman presented with a 12 month history of gradually worsening painless involuntary flexion of the left middle and ring fingers associated with some tightness of the left forearm and occasional paraesthesia in the fingertips. Her symptoms increased until her fingers were fully and forcefully flexed against her palm. She also developed similar but milder symptoms in the right hand. Most manual functions apart from pinch grip were impaired. The abnormal finger posture persisted during sleep.

She had severe chronic obstructive pulmonary disease (COPD) and was taking regular nebulised salbutamol (up to $5 \mathrm{mg}$ four times daily), oxygen, and prednisolone.

Examination showed continuous involuntary flexion of the middle and ring fingers at the metacarpophalangeal and the proximal inter- 
Figure 2 EMG in the right flexor digitorum superficialis muscle of patient 1. (A) A spontaneous high frequency neuromyotonic discharge is shown with the characteristic gradually waning amplitude. Similar discharges were continuously present in the left side without any pause. (B) A small tap on the median nerve at the elbow produces a similar discharge (vertical bar $=1 \mathrm{mV}$, horizontal bar $=500 \mathrm{~ms}$ ).

phalangeal joints (fig 1). There was some stiffness but no hypertrophy, myokymia, or percussion myotonia of the forearm muscles. There were no other neurological abnormalities; in particular power and reflexes were normal.

\section{Case 2}

A 70 year old woman, also with severe COPD and receiving home nebulised salbutamol $(2.5$ $\mathrm{mg}$ four times daily) and oxygen, presented with a 10 month history of progressive painless flexion of the left middle and ring fingers with similar but much milder symptoms in the right hand. She had cramps in the left forearm and difficulty writing and feeding herself because of the abnormal finger posture.

Examination confirmed involuntary flexion of the left middle and ring fingers (fig 1). There was also mild weakness of left finger abduction. Again there was no muscle hypertrophy, myokymia, percussion myotonia, or any other abnormal neurological findings.

INVESTIGATIONS

Routine blood tests including full blood count; liver, renal, and thyroid function tests; serum calcium, phosphate, protein electrophoresis and erythrocyte sedimention rate were normal. Autoantibody screen including rheumatoid factor and antibodies to GM1, glutamic acid decarboxylase, and voltage gated potassium channels were negative. Blood gases were normal on oxygen but hypoxaemia was repeatedly documented during acute exacerbation of their pulmonary disease. Recent chest radiography showed no active changes.

Their severe COPD prevented cranial or cervical imaging, CSF examination, and more elaborate neurophysiological studies such as regional nerve block and curarisation.

The motor conduction velocities and $\mathrm{F}$ wave latencies in both median and ulnar and right lateral and medial popliteal nerves were normal. Stimulation of median and ulnar nerves at the elbow and Erb's point (while recording over relevant hand muscles or flexor digitorum superficialis muscles at the midforearm) did not show any significant reduction in the amplitude of compound muscle action potentials or slowing of conduction. Sensory studies in both median and ulnar, left or right radial, and sural nerves were normal except for evidence of a mild subclinical bilateral carpal tunnel syndrome in patient 2 . 
Electromyography with a concentric needle electrode in the flexor digitorum superficialis muscles in both patients showed continuous motor unit discharges at high rate $(70-300 \mathrm{~Hz})$. The discharges stopped at times spontaneously to reappear within a few seconds but more often they continued for several minutes, gradually waning in amplitude (fig 2). They were particularly continuous in the left flexor digitorum superficialis muscle. Similar discharges were noted in the left extensor digitorum communis and biceps muscles of patient 2 only. The morphology of motor unit potentials was normal or mildly polyphasic. Interference pattern was full. These discharges were not seen in the left flexor carpi ulnaris, first dorsal interosseous, or the right tibialis anterior or vastus medialis muscles which were examined on different occasions during the follow up period.

In patient 1 , a trial of carbamazepine and phenytoin was ineffective. After an injection of 200 units of botulinum toxin (Dysport) into the left flexor digitorum superficialis muscle (under EMG guidance) the fingers could be held at their fully extended limit. A repeat EMG study 1 week later showed no spontaneous motor unit discharges but a neurogenic pattern with reduced interference pattern. Unfortunately, this injection weakened her pinch grip, such that she could no longer open her salbutamol capsules and she declined further injections. Patient 2 declined any treatment. Both patients were supplied with a special splint and their condition has remained static over an 18 month follow up period.

\section{Discussion}

These two remarkably similar cases have spontaneous discharges in selected upper limb muscles with features of neuromyotonia, which were unusually continuous, leading to involuntary flexion and contracture of their ring and middle fingers.

Neuromyotonia is a clinicoelectromyographic syndrome. ${ }^{3-5}$ Clinically the main feature is muscle stiffness often associated with cramps, myokymia, and slow relaxation. Fasciculations, muscle hypertrophy, paraesthesia, excessive perspiration, and, rarely, pain and abnormal limb posture may be present. The main neurophysiological feature is the presence of distinctive neuromyotonic discharges which are high frequency motor unit discharges, occurring in bursts or almost continuously, beginning and ending abruptly or slowly waning in amplitude with a special (pinging) sound on EMG. Myokymic discharges may be present and if predominant the term myokymia is often used. ${ }^{5}$ Myotonic discharges can be differentiated from neuromyotonic discharges by their specific "dive bomber" sound due to waxing and waning in their discharge frequency. Complex repetitive (bizarre high frequency) discharges, seen in various neurogenic and myopathic disorders, have very complex motor unit potentials firing at a lower frequency, and are not associated with clinical features of neuromyotonia. ${ }^{3}$
Neuromyotonic (and myokymic) discharges are generated by an unstable segment of axon membrane, distally or proximally, spontaneously or evoked by traversing impulses (autoexcitation or ephaptic). ${ }^{58}$ They are characteristically not affected by general or spinal anaesthesia, but are completely abolished by neuromuscular blocking agents and diminished to a variable degree by nerve block depending on the site of generation. ${ }^{34} 79$

Generalised neuromyotonia which is sometimes associated with peripheral neuropathy has been used synonymously with Isaacs' syndome,${ }^{10}$ initially referred to as "the syndrome of continuous muscle fibre activity". An autoimmune aetiology for generalised neuromyotonia has been implicated by the presence of oligoclonal bands in the CSF, association with other autoimmune diseases, improvement after plasma exchange and most recently with the detection of antibodies directed against voltage gated potassium channels. ${ }^{1011}$ It is postulated that the down regulation of fast potassium channels may prolong depolarisation, leading to the repetitive firing of motor units. ${ }^{10}$ An autoimmune pathogenesis has also been suggested for neuromyotonia associated with malignancies, ${ }^{12}{ }^{13}$ autoimmune neuropathies, and penicillamine. ${ }^{14} 15$

Generalised neuromyotonia may present with abnormal posture of limbs but neurophysiological evidence of more widespread pathology is usually present. ${ }^{2}$

Focal neuromyotonia/myokymia is seen after radiotherapy, ${ }^{4}$ in multifocal motor neuropathy, ${ }^{16}$ and in transposed muscle flaps. ${ }^{17}{ }^{18}$ Neuromyotonic discharges may be seen with mechanical irritation of the nerves during surgery. ${ }^{3}$ Focal myokymia is reported in chronic radiculopathies, after nerve injury, and, rarely, with compression neuropathies. ${ }^{4}$ Hyperexcitability after axonal injury is primarily due to local remodelling of voltage gated cation channels which may be expressed by spontaneous ectopic firing and hypersensitivity to hypoxic, chemical, or other stimuli. Both increased sodium and decreased potassium conductance may lead to repetitive firing. ${ }^{19}$

Although the selective involvement of C6-C8 innervated muscles bilaterally with normal nerve conduction studies is suggestive of root pathology, imaging studies were not possible to investigate this further. However, this alone would not explain the abnormalities found. Both the advanced COPD and chronic use of salbutamol may be relevant.

Ischaemia may increase or decrease neuromyotonia. ${ }^{202}$ Acute hypoxia produces hyperpolarisation but at a later stage or after prolonged insult axons fire ectopically, in high frequency bursts. The most proximal part of the nerve is more liable to fire repetitively. ${ }^{21}$ Hyperventilation leading to decreased serum ionised calcium and alkalosis enhances neuromyotonia. ${ }^{22}$ In healthy subjects hypocalcaemia and ischaemia produce spasms with repetitive firing of motor units. ${ }^{23}$ The mechanism of ischaemia induced spasm is probably different from neuromyotonia although they both could be present in the same patient. ${ }^{24}$ 
Salbutamol activates sodium-potassium transport in skeletal muscle producing hyperpolarisation and hypokalaemia but it is not clear if a similar effect is seen in the nerve membrane and if this leads to hyperexcitability at a later stage. $^{25}$

The selective flexion of just the middle and ring fingers is curious because the neuromyotonic discharges affect the whole of flexor digitorum superficialis muscle. There are two possible anatomical explanations. ${ }^{26}$ Firstly, the additional extensor muscles (extensor digiti minimi and extensor indices) of the little and index finger may prevent their involuntary flexion. Secondly the flexor digitorum superficialis muscle fibres are divided into superficial and deep layers ending in the tendons of ring and middle, and index and little fingers, respectively. There is a similar arrangement for their tendons behind the flexor retinaculum. It is tempting to speculate that this superficial location of the flexor muscle fibres and tendons may make them more susceptible to irritation, enhancing the clinical manifestation of neuromyotonia in the middle and ring fingers. An awareness of this anatomical division during botulinum toxin injection in the flexor digitorum superficialis muscles is important because if the neuromuscular blockade involves the whole muscle, although the flexion deformity improves, the resulting weakness of the index flexor causes weakness of pinch grip and increased functional disability. This may be avoidable if the injection is given, in small dosage, in the superficial layer of the flexor digitorum superficialis muscles sparing the index and little finger flexors.

The extraordinary similarity of our two patients suggests a common aetiology. We think that this is a novel form of focal neuromyotonia probably confined to elderly people. A combination of factors such as local nerve root damage, chronic hypoxia, and salbutamol use may be contributing. We are uncertain if these two patients are a rarity or whether some similar patients could be found incorrectly diagnosed as having Dupuytren's contracture. In elderly patients with unexplained abnormal finger posture, focal neuromyotonia needs to be considered.

1 Marsden CD, Obeso JA, Traub MM, et al. Muscle spasms associated with Sudeck's atrophy after injury. $B M \mathcal{F}$ 1984;288:173-6.
2 Auger RG, Daube JR, Gomez MR, et al. Hereditary form of sustained muscle activity of peripheral origin causing generalised myokymia and muscle stiffness. Ann Neurol 1984: 15:13-21.

3 Daube JR. AAEM minimonograph 11: Needle examination in clinical electromyography. Muscle Nerve 1991;14:685-70

4 Sivak M, Ochoa J, Fernandez JM. Positive manifestation of nerve fibre dysfunction: clinical electrophysiologic and pathologic correlates. In: Brown WF, Bolton CF, eds. Clinical electromyography. 2nd ed. Boston:ButterworthHeinemann 1993:134-5.

5 Layzer RB. Neuromyotonia: a new autoimmune disease. Ann Neurol 1995;38:701.

6 Hosokawa S, Shinoda H, Sakai T, et al. Electrophysiological study on limb myokymia in three women. I Neurol Neurosurg Psychiatry 1987;50:877-81.

7 Guttman L. AAEM minimonograph 37: facial and limb myokymia. Muscle Nerve 1991;14:1043-9.

8 Tobergsen T, Stalberg E, Brautaset NJ. Generator sites for spontaneous activity in neuromyotonia. An EMG study. Electroencephalogr Clin Neurophysiol 1996;101:69-78.

9 Isaacs H. A syndrome of continuous muscle-fibre activity. 7 Neurol Neurosurg Psychiatry 1961;24:319-25.

10 Newsom-Davis J, Mills KR. Immunological associations of neuromyotonia (Isaacs' syndrome). Report of five cases and review of the literature. Brain 1993;116:453-69.

11 Hart IK, Waters C, Vincent A, et al. Autoantibodies detected to expressed $\mathrm{K}^{+}$channels are implicated in neuromyotonia. Ann Neurol 1997;41:238-46.

12 Caress JB, Abend WK, Preston DC, et al. A case of Hodgkin's lymphoma producing neuromyotonia. Neurology 1997;49:258-9

13 Partanen VSJ, Soininen H, Saksa M, et al. Electromyographic and nerve conduction findings in a patient with neuromyotonia, normocalcemic tetany and small-cell lung cancer. Acta Neurol Scand 1980;61:216-26.

14 Vasilescu C, Alexianu M, Dan A. Muscle hypertrophy and a syndrome of continuous motor unit activity in prednisolone: responsive Guillain-Barre polyneuropathy. 7 Neurol 1984;231:276-9.

15 Reeback J, Benton S, Swash M, et al. Penicillamine induced neuromyotonia. BMF 1979;1:1464-5.

16 Thomas PK, Claus D, Jaspert A, et al. Focal upper limb demyelinating neuropathy. Brain 1996;119:765-74.

17 Samuel M, Manford M, Sheean G, et al. A neuromyotonic process in a transposed latissimus dorsi muscle stemming from radiotherapy and peripheral surgery. Mov Disord 1996;11:449-51

18 Schwartz MS, Wren DR, Filshie J. Neuromyotonia in a muscle flap producing a convulsing breast: successful treatment with botulinum toxin. Mov Dis 1998;13:188-90.

19 Devor M. Abnormal excitability in injured axons. In: Waxman SG, Kocsis JD, Stys PK, eds. The axon. New York: Oxford University Press 1995:530-52.

20 Lance JW, Burk D, Pollard J. Hyperexcitability of motor and sensory neurons in neuromyotonia. Ann Neurol 1979;5: 523-32.

21 Bostock H, Baker M, Reid G. Changes in excitability of human motor axons underlying post-ischemic fasciculations: evidence for two stable states. I Physiol 1991;441:537-57

22 Brick J F, Gutmann L, McComas C F. Calcium effect on generation and amplification of myokymic discharges. Neurology 1982;32:618-22.

23 Kugelberg E. Activation of human nerves by hyperventilation and hypocalcemia. Arch Neurol Psychiatry 1948;60: $153-64$.

24 Oda K, Fukushima N, Shibasaki H, et al. Hypoxia-sensitive hyperexcitability of the intramuscular nerve axons in Isaac' syndrome. Ann Neurol 1989;25:140-5.

25 Clausen T. Regulation of active $\mathrm{Na}^{+}-\mathrm{K}^{+}$transport in skeletal muscle. Physiol Rev 1986;66:542-80

26 Williams P L, Warwick R, Dyson M, et al. Gray's anatomy. 37th ed. London: Churchill Livingston, 1989:617-31. 